\title{
Dynamic Random Scan Approach of Spectrum Imaging for Temporal Evolution of Spectroscopic Signals
}

\author{
Alberto Zobelli ${ }^{1}$, Steffi Y. Woo ${ }^{1}$, Luiz H.G. Tizei ${ }^{1}$, Nathalie Brun ${ }^{1}$, Xiaoyan Li $^{1}$, Anna Tararan ${ }^{1}$, Odile \\ Stéphan ${ }^{1}$, Mathieu Kociak ${ }^{1}$ and Marcel Tencé ${ }^{1}$ \\ 1. Laboratoire de Physique des Solides, Université Paris-Sud, CNRS, Orsay, France \\ * Corresponding author: alberto.zobelli@u-psud.fr
}

The evolution of the scanning modules for scanning transmission electron microscopes (STEM) now makes possible to generate arbitrary scanning patterns, and to approach scanning modes already used in other techniques. The typical limited scanning speed of the probe displacements, with circuits based on magnetic coils, does not necessarily warrant its use in bright-field and annular dark-field (BF/ADF) image modes. This limit does not apply in hyperspectral imaging for which longer pixel dwell-times are required. The use of a randomized sequence of the scanning matrix with a fast beam-blanker can limit dose accumulation effects when investigating irradiation sensitive materials. Unlike standard sequential scanning modes, a pre-defined pattern with fully shuffled raster order can sample the entire frame homogeneously in a given time window, such that the time evolution of the structural and spectroscopic signal can also be followed. Furthermore, inpainting processes can be applied to reconstruct the full image from partial sparse image sets [1]. With regard to sample drift correction by frame averaging [2], a series of reconstructed images obtained with the same pixel number at successive time intervals can provide any structural plus spectral changes occurring within the full hyperspectral image acquisition time [3]. In this contribution, the benefits of using this special randomized scanning mode for electron energy-loss spectroscopy (EELS) and cathodoluminescence (CL) experiments in a STEM is demonstrated, and how it enables to recover temporal information, which would be otherwise not feasible.

With conventional sequential scan, in addition to the time and space domains being coupled, highly beam sensitive emitters such as molecules or defects can accumulate damage and suddenly blink or bleach [Fig. 1(d)]. Using random scan, pixels are distributed homogeneously over the entire area containing emitters such that sufficient CL signal can be obtained before eventual direct impingement and bleaching, demonstrated by the symmetric spatial extension of the signal intensity [Fig. 1(c)]. With the pixel raster order known, it is possible to reconstruct a time sequence of inpainted images from a segment of the total acquired pixels to track spectroscopic changes [Fig. 1(d)]. Specimen drift in atomically-resolved EELS mapping can be corrected in a similar manner with random scan, by applying shifts from the alignment of the ADF image pair on generated elementals maps [Fig. 2] or alternatively to the datacube to recover the full spectral channels, and supplemented by subsequent inpainting. Moreover, live inpainting of the most recently acquired pixels during data acquisition further extend the capabilities of random scan to monitor the dynamics of spectroscopic signals with a fraction of the total dose and acquisition time [4].

\section{References:}

[1] A Stevens et al., Microscoopy 63 (2014), p. 41.

[2] L Jones et al., Microscopy 67 (2018), p. i98.

[3] A Zobelli et al., in preparation.

[4] The authors acknowledge funding from the ANR, program of future investment TEMPOSCHROMATEM (No. ANR-10-EQPX- 50). SYW acknowledges NSERC for the fellowship funding. 

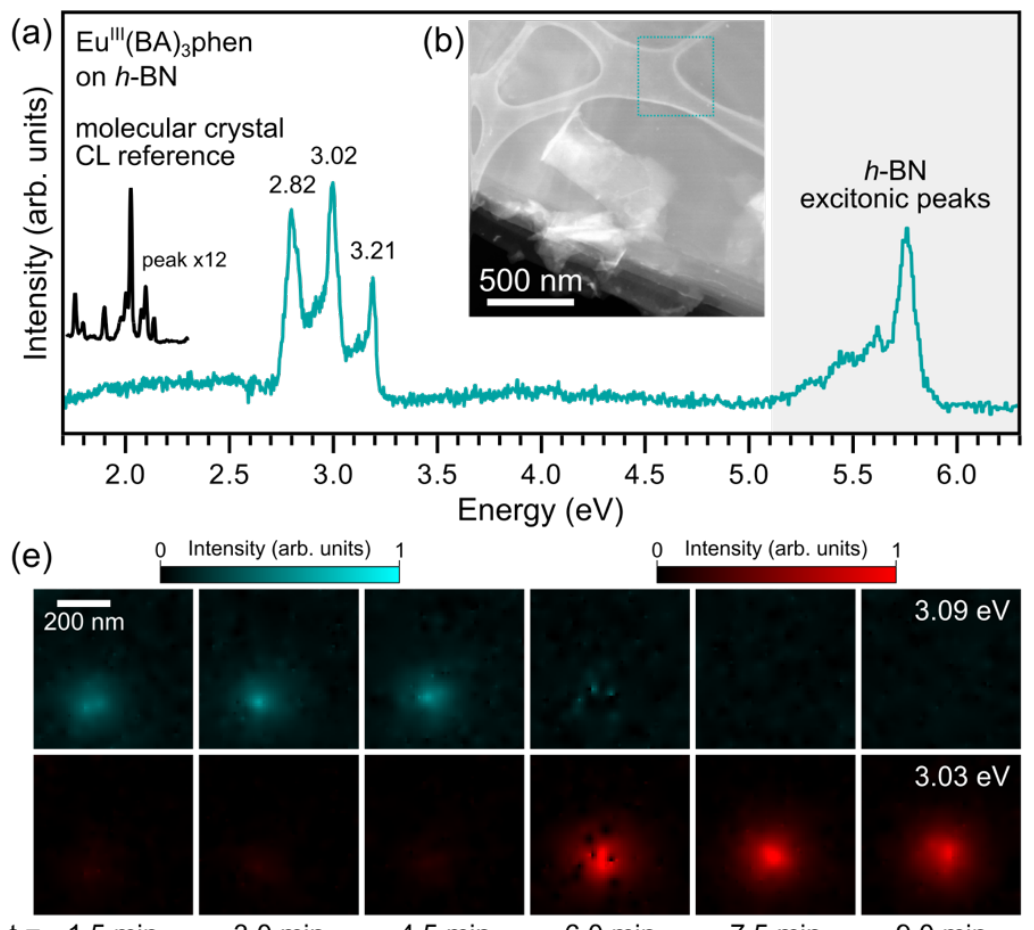

$\mathrm{t}=1.5 \mathrm{~min}$

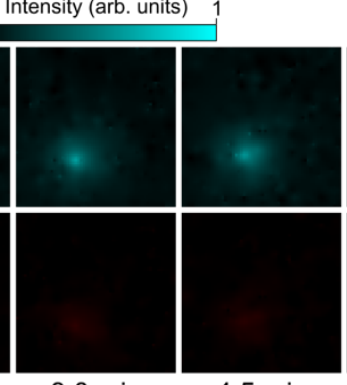

$3.0 \mathrm{~min}$

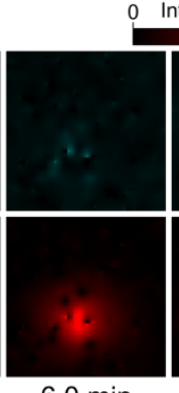

$6.0 \mathrm{~min}$

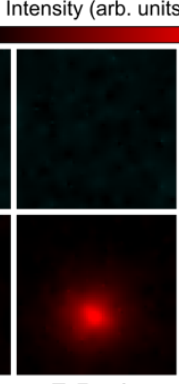

$7.5 \mathrm{~min}$ (c) random scan

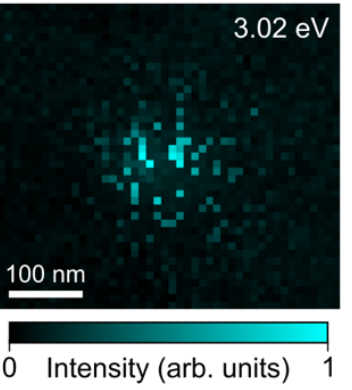

(f)

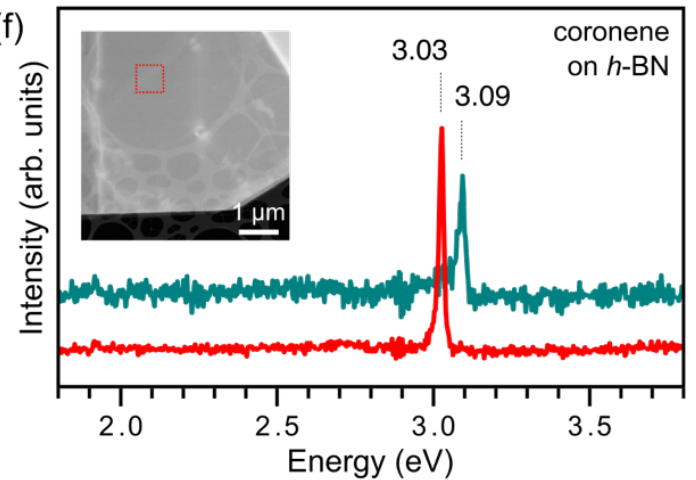

(d) sequential scan
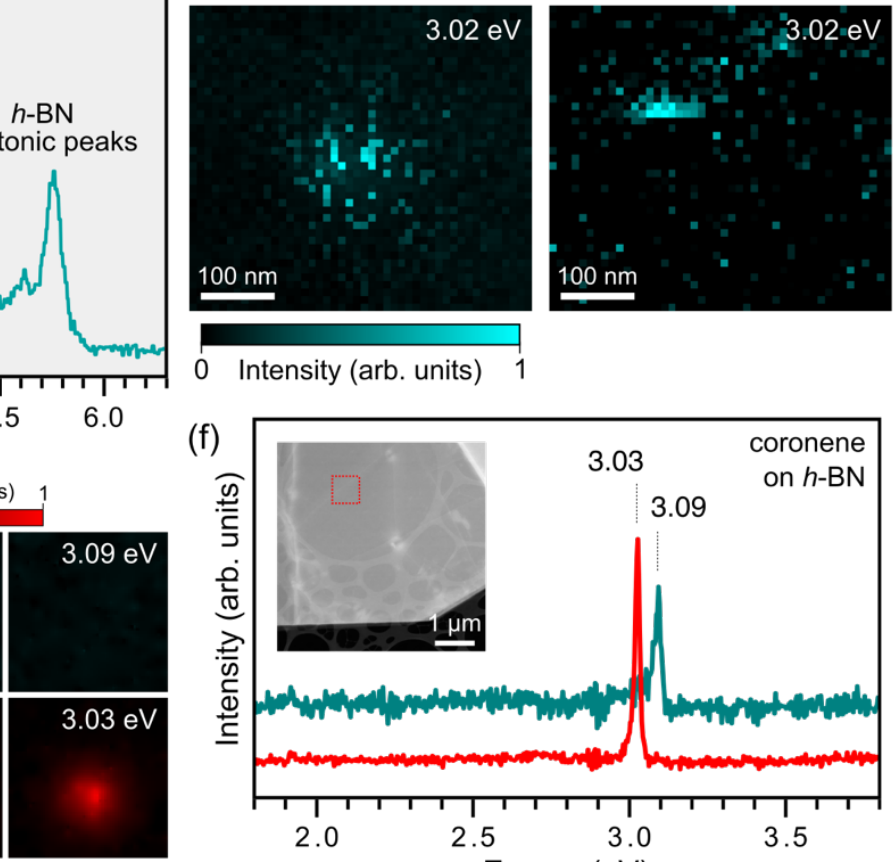

$9.0 \mathrm{~min}$

Figure 1. $\mathrm{CL}$ spectrum imaging of molecules, Eu-complexes (a-d) and coronene (e,f), on $h$-BN: comparing CL intensity maps of emission in (a) obtained using (c) random scan and (d) conventional sequential scan of the same region. (e) Time-sequenced inpainted CL maps of the emissions in (f) from segmentation of the random-scanned spectrum image demonstrating a transition at $\sim 6$ min.
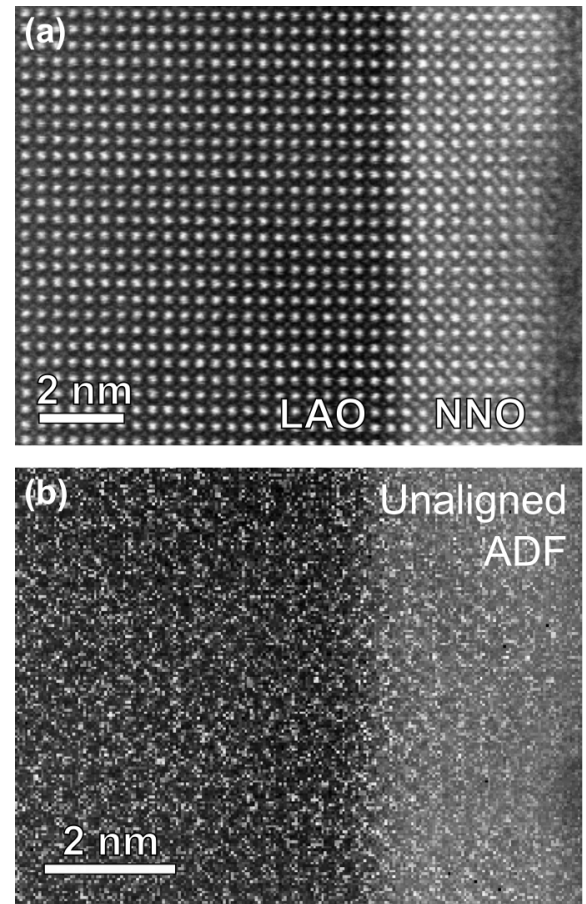
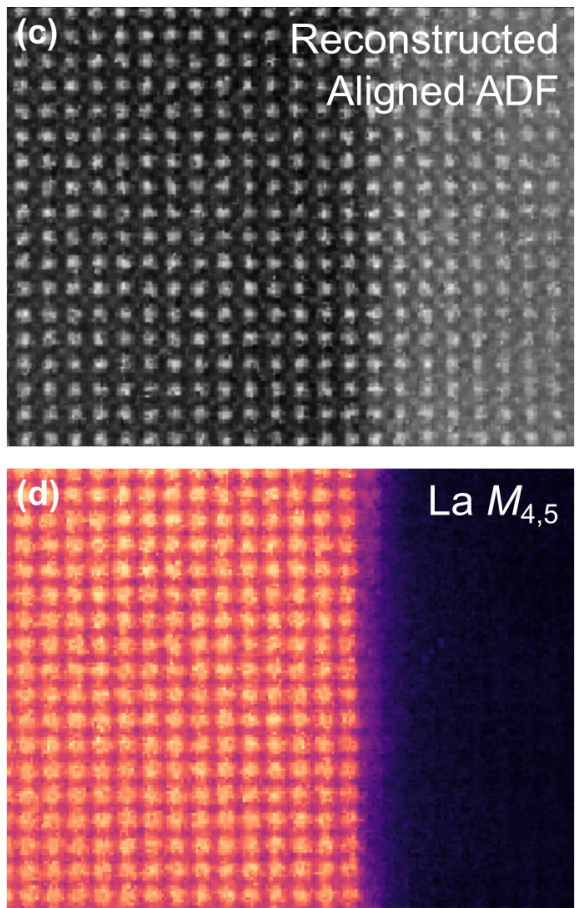
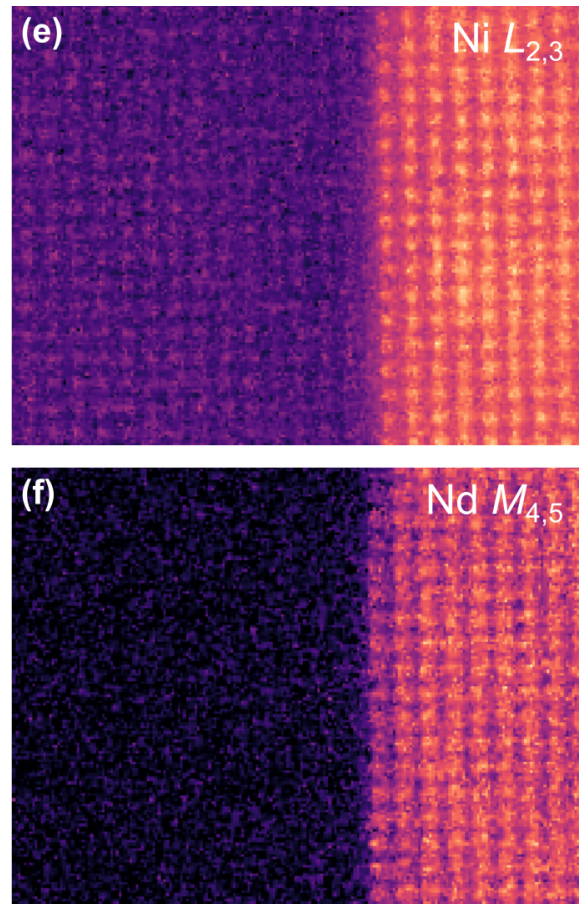

Figure 2. $\mathrm{NdNiO}_{3}$ (NNO) thin film grown on $\mathrm{LaAlO}_{3}$ (LAO) substrate: atomically-resolved EELS mapping with random scan, showing the concurrent ADF (b) before and (c) after drift correction, and (df) the resulting inpainted elemental maps. 